\title{
Update sul management e trattamento del paziente con lesioni cutanee croniche
}

\author{
Aurora Parodi, ${ }^{1}$ Valeria Maria Messina, ${ }^{2}$ Manuela Martolini, ${ }^{3}$ Shpresa Haxhiaj, ${ }^{4}$ Emanuele Claudio Cozzani ${ }^{1}$ \\ ${ }^{1}$ DiSSal -Dipartimento di Scienze della Salute Università di Genova-S.C. Clinica Dermatologica IRCCS Ospedale Policlinico San \\ Martino, Genova; ${ }^{2}$ Ambulatorio Convenzionato ASL 3 Genovese, Genova; ${ }^{3}$ Dipartimento Cure Primarie ASL 2 Savonese, Savona; \\ ${ }^{4}$ Assistenza Domiciliare distretto Finale Ligure, ASL 2 Savonese, Savona, Italia
}

\section{RIASSUNTO}

Le lesioni da decubito, le ulcere vascolari e il piede diabetico rappresentano le lesioni cutanee croniche maggiormente diffuse in età geriatrica. La lesione cutanea cronica presenta un elevato rischio infettivo ed un management complesso che richiede terapie mirate ed un iter di trattamento specifico. Il metodo TIME (Tissue management control of Infection and inflammation Moisture imbalance advancement of the Epithelial edge of the wound), costituisce il gold standard per il trattamento delle lesioni cutanee croniche poiché

Corrispondenza: Aurora Parodi, DiSSal Clinica Dermatologica Ospedale Policlinico San Martino, Genova, Via Balbi 5, 16126 Genova (GE), Italy.

E-mail: aurora.parodi40@gmail.com

Key words: Lesioni croniche; ipoclorito di sodio; telemedicina.

Ringraziamenti: Gli autori ringraziano Claudia Laterza, MD di Sanitanova s.r.l., e Michela Clemente per le attività di medical writing e assistenza editoriale. Gli autori ringraziano anche Health Search, unità di ricerca della Società Italiana di Medicina Generale e delle Cure Primarie (S.I.M.G.), per la condivisione dei dati (tutti i diritti riservati).

Finanziamento: Questo lavoro è stato realizzato grazie al contributo non condizionante di Angelini S.p.A.

Conflitti di interesse: AP è consulente per Abbvie, Almirall, Novartis, Pfizer, Leo pharma, Lilly, Galderma, Angelini, Celgene, Alfasigma. Gli altri autori non dichiarano conflitti di interesse.

Contributo degli autori: AP ha dato un contributo sostanziale all'ideazione e alla progettazione del manoscritto. Tutti gli autori hanno partecipato alla stesura del manoscritto e lo hanno rivisto criticamente. Tutti gli autori hanno letto e approvato la versione finale del manoscritto. Gli autori non segnalano alcun coinvolgimento nella ricerca da parte dello sponsor che possa aver influenzato il risultato di questo lavoro.

Disponibilità di dati e materiali: Tutti i dati analizzati in questo studio sono disponibili nel presente articolo.

Approvazione etica e consenso alla partecipazione: Non applicabile.

Consenso alla pubblicazione: Non applicabile.

Ricevuto per la pubblicazione: 29 giugno 2021.

Revisione ricevuta: 29 novembre 2021.

Accettato per la pubblicazione: 7 dicembre 2021.

This work is licensed under a Creative Commons Attribution NonCommercial 4.0 License (CC BY-NC 4.0).

${ }^{\circ}$ Copyright: the Author(s), 2021

Licensee PAGEPress, Italy

Italian Journal of Wound Care 2021; 5(3):78

doi:10.4081/ijwc.2021.78 consente di controllare l'infezione e il grado di macerazione della ferita anche nei margini epiteliali. La soluzione di ipoclorito di sodio alla concentrazione dello $0,05 \%$, oggetto di numerosi studi in letteratura, grazie ad un ampio spettro germicida ed alla sua elevata compatibilità tissutale rappresenta il metodo di disinfezione d'elezione per il trattamento delle lesioni cutanee croniche. La qualità di vita (Quality of Life, QoL) di un paziente affetto da lesione cutanea cronica può essere fortemente compromessa. La formazione di un team sanitario multidisciplinare per la gestione del patient journey può favorire il percorso di guarigione, facilitare la gestione della lesione nella quotidianità e migliorare la QoL del paziente. La telemedicina spicca tra le modalità innovative di gestione del wound care sperimentate da un'equipe di specialisti del territorio ligure negli ultimi mesi a seguito della pandemia COVID-19. La pratica della telemedicina si è rivelata particolarmente utile nel follow up della lesione cronica a fronte di un adeguato impiego di strumenti tecnologici che permettano un'elevata qualità di immagini.

\section{LESIONI CUTANEE CRONICHE: EPIDEMIOLOGIA E PRINCIPALI CARATTERISTICHE}

Le lesioni cutanee croniche costituiscono una delle patologie più frequenti dell'età geriatrica e, considerato il costante incremento dell'età anagrafica media della popolazione italiana ed europea, incidono fortemente sia a livello sociale che economico. ${ }^{1}$

In Italia si stima che vi siano circa 2 milioni di individui affetti da lesioni cutanee croniche.

In una delle regioni italiane come la Liguria, eletta modello europeo per la longevità della popolazione, un terzo degli abitanti ha almeno 60 anni con un trend in aumento per quanto concerne i residenti centenari (3,3 abitanti centenari per 100.000). ${ }^{2} \mathrm{Nel} 2018$ in Liguria, secondo Health Search, si contano 5.666 pazienti con lesioni cutanee croniche con forte prevalenza del sesso femminile.

Le lesioni cutanee croniche maggiormente frequenti 
in età avanzata sono: ulcere da pressione o lesioni da decubito, ulcere vascolari e piede diabetico. ${ }^{3,4}$

Le lesioni da decubito sono causate da una pressione costante e prolungata sulla cute. ${ }^{5}$ Le sedi tipiche delle ulcere da decubito sono l'area dorso glutea, l'area sacrale e l'area del calcagno che rappresentano le zone di maggior contatto del corpo nei soggetti allettati o con ridotta mobilità. La scarsa ossigenazione tissutale, l'elevata compressione dei vasi sanguigni ed un alto grado di umidità compromettono l'integrità dell'epidermide e del derma fino a determinare l'insorgenza di ulcere cutanee favorite da condizioni legate allo status del paziente come la malnutrizione, il diabete, l'incontinenza e i disturbi neuro-psichici.

Il trattamento di queste lesioni si basa essenzialmente su interventi tesi a migliorare le condizioni generali e nutrizionali del soggetto, sulla riduzione della pressione locale grazie ad un costante riposizionamento del paziente e su un adeguato drenaggio della zona lesa unitamente allo sbrigliamento tissutale onde favorire una nuova granulazione del tessuto. Per la detersione e la pulizia dell'ulcera da pressione possono essere impiegati diversi antisettici locali, al fine di limitare la proliferazione batterica e consentire la guarigione tissutale. Le tipologie di medicazione adatte a questo tipo di lesione sono molteplici ${ }^{6}$ e la scelta della medicazione si basa sulla sede della lesione (es. sacrale, arto inferiore ecc), dimensione della ferita e qualità e quantità di essudato prodotto. ${ }^{7,8}$

Le lesioni cutanee vascolari croniche si localizzano in particolare agli arti inferiori e presentano un'eziologia di tipo vascolare, venosa o arteriosa. ${ }^{3}$ La maggior parte delle lesioni cutanee ad origine vascolare vengono associate a morbilità vascolari in prevalenza venose (fino al $70 \%$ ) ed in minor parte arteriose (fino al 10\%). In alcuni casi si possono riscontrare lesioni cutanee ad eziologia mista (venosa/arteriosa). Tra le maggiori cause delle lesioni cutanee vascolari croniche troviamo le arteriopatie, l'insufficienza venosa cronica, le vasculiti, e il linfedema.

Il trattamento d'elezione in caso di lesioni cutanee croniche di tipo venoso consiste nella terapia compressiva attraverso l'impiego di una compressione elevata (30-40 mmHg alla caviglia). ${ }^{9}$ Nei pazienti con difficoltà di deambulazione è preferibile l'impiego di bendaggi elastici e bendaggi multistrato. Nei soggetti affetti da ulcere a eziologia mista (A/V), si consiglia l'impiego di un trattamento compressivo ridotto. ${ }^{10}$

Il trattamento delle lesioni cutanee arteriose croniche si avvale della correzione chirurgica e/o dell'utilizzo di terapie farmacologiche per l'insufficienza vascolare.

Il piede diabetico ${ }^{11}$ rappresenta una delle complicanze più gravi della patologia diabetica. L'indice di prevalenza dell'ulcerazione del piede nei soggetti diabetici è del 4$10 \%$, con una netta maggioranza di pazienti anziani. ${ }^{12,13}$ Le ulcere sono dovute a due fattori: alterazioni arteriose profonde e neuropatia. ${ }^{14}$ Il rischio di complicanze gravi quali l'amputazione del piede è alto e fortemente legato all'età del paziente diabetico. La neuropatia diabetica è presente in circa il $90 \%{ }^{11}$ delle ulcerazioni del piede diabetico e, unitamente alla patologia arteriosa e ai traumi del piede, costituisce uno dei principali fattori di rischio delle ulcere del piede diabetico. La conseguente scarsa integrità della pelle è il background ideale per lo sviluppo di infezioni microbiche. Il trattamento delle lesioni cutanee croniche del piede diabetico si concentra su un'adeguata detersione e antisepsi della ferita con eventuali procedure di rivascolarizzazione se indicate.

Lo sbrigliamento chirurgico della ferita rappresenta il trattamento gold standard per le ulcere diabetiche dopo un eventuale intervento di rivascolarizzazione. ${ }^{15}$

\section{APPROCCIO CLINICO DELLA LESIONE CUTANEA CRONICA: DETERSIONE, DEBRIDEMENT E MEDICAZIONI}

La lesione cronica è una lesione complessa di difficile guarigione, a elevato rischio infettivo che richiede l'adozione di terapie efficaci e di un team di cura multidisciplinare. ${ }^{16}$ I tre step fondamentali nel trattamento di una lesione cutanea sono detersione, debridement e medicazione. Il WBP (Wound Bed Preparation) ovvero la preparazione ottimale del letto della ferita rappresenta un aspetto fondamentale nella gestione della ferita cronica caratterizzata da una flogosi elevata e da un insufficiente processo riparativo. ${ }^{17}$ Le ulcere croniche hanno spesso un'alta incidenza di biofilm (aggregato di microrganismi inglobati in una matrice di sostanze polimeriche extracellulari), che porta ad una risposta infiammatoria persistente, proteolisi eccessiva, e degradazione dei fattori critici di crescita e della matrice extracellulare. Il PH alcalino caratteristico delle ulcere croniche favorisce l'ulteriore proliferazione batterica ${ }^{18,19}$ e ritarda la riepitelizzazione e la guarigione della ferita.

Nel 2002 un'equipe di esperti ${ }^{17}$ ha sviluppato il metodo TIME (Tissue management control of Infection and inflammation Moisture imbalance advancement of the Epithelial edge of the wound) ovvero trattamento del tessuto non vitale, controllo dell'infezione, controllo della macerazione, controllo dei margini epiteliali, che rappresenta una guida per la gestione dei pazienti con lesioni cutanee croniche.

La detersione primaria della lesione attraverso il lavaggio della stessa è mirato a rimuovere meccanicamente diverse tipologie di materiale presente nella ferita, tra cui tessuto devitalizzato, detriti e microbi. Gli Autori raccomandano di detergere anche i margini e la cute perilesionale fino a $15-20 \mathrm{~cm}$ e non solo il centro della ferita per migliorare gli outcome clinici.

Successivamente è necessario agire con un antisettico topico per prevenire o eliminare la proliferazione batte- 
rica. ${ }^{20}$ Le popolazioni microbiche riscontrate con maggior frequenza nelle lesioni cutanee appartengono a diverse categorie: Gram positivi (Staphilococcus Aureus), Gram negativi (Pseudomonas Aeruginosa, Proteus Mirabilis, Acinetobacter), lieviti (Candida Albicans). L'European Wound Management Association e la British Society for Antimicrobial Chemotherapy raccomandano, per il trattamento delle lesioni cutanee croniche, l'uso preferenziale di antimicrobici topici non contenenti antibiotici. ${ }^{21-23}$

La scelta dell'antisettico da impiegare dipende dalle sue caratteristiche chimiche e fisiche e dalle indicazioni: nella scelta vanno considerati diversi fattori quali la citossicità, l'istocompatibilità, la eventualità di provocare allergie o dolore, la possibilità di interazione con altre sostanze e l'efficacia nel diminuire la carica microbica sia superficiale che profonda senza determinare resistenze. ${ }^{24}$

Gli antisettici che esplicano un'azione rapida e ad ampio spettro microbico sono gli alogenati (derivati di cloro e iodio); in particolare i derivati del cloro possiedono un'elevata efficacia unita ad un'elevata istocompatibilità, motivo per cui sono ben tollerati dai tessuti. ${ }^{25}$

Gli antisettici generalmente utilizzati per la detersione della cute lesa sono: i) iodopovidone (concentrazione $10 \%$ ); ii) clorexidina (concentrazione $0.5 \%$ ); iii) sodio ipoclorito (concentrazione $0.05 \%$ ).

Lo iodopovidone possiede un ampio spettro germicida ed è efficace su batteri Gram-positivi e negativi, miceti e virus. ${ }^{26}$ Lascia una colorazione giallo-arancione sull'epidermide ad ogni utilizzo e la sua azione non immediata costituisce un possibile gap nella gestione dell'antisepsi di ferite croniche; inoltre deve essere conservato al riparo dalla luce e dal calore. ${ }^{27}$ Lo iodopovidone può causare alterazioni della funzione tiroidea in caso di trattamenti prolungati su aree vaste.

La clorexidina possiede un potere biocida più limitato, e un $\mathrm{pH}$ ottimale per sua attività tra 5 e 7 . Può provocare dermatiti eczematose da contatto in soggetti sensibili.

La soluzione di sodio ipoclorito alla concentrazione $0.05 \%$ è una soluzione isotonica stabile che, oltre all'ampio spettro germicida su batteri Gram-positivi (anche MRSA, Staphylococco aureo meticillino resistente) e negativi, spore e virus, presenta un elevato grado di istocompatibilità che la rende trattamento d'elezione nella disinfezione dei tessuti lesi ${ }^{28}$ avendo dimostrato di non ostacolare il processo di riepitelizzazione anche confrontata con il trattamento a base di soluzione fisiologica. ${ }^{25,29,30}$ Inoltre, la combinazione di $\mathrm{PH}$ acido e alto potenziale ossidoriduttivo inibiscono le metalloproteasi nel microambiente di ferita accelerando i tempi di riparazione.

Rispetto allo iodopovidone, non colora l'epidermide, agisce in tempi più rapidi e non ha effetti sistemici, mentre ha una maggiore efficacia antisettica e tollerabilità tissutale rispetto alla clorexidina.

Il debridement (letteralmente "sbrigliamento") della ferita rappresenta un altro step fondamentale del trattamento di una lesione cutanea cronica. Questo processo è mirato alla rimozione del tessuto necrotico presente nella ferita e può essere praticato più volte sulla stessa lesione ${ }^{31}$ Lo sbrigliamento tissutale è necessario anche per diminuire la carica batterica all'interno della lesione. Esistono diversi tipi di debridement tra cui il debridement chirurgico, il debridement autolitico, il debridement enzimatico e il debridement biologico. ${ }^{32,33}$ Il debridement autolitico è così denominato poiché è un processo endogeno supportato da un ambiente umido, grazie al quale fagociti ed enzimi proteolitici degradano il tessuto necrotico. Attualmente sul mercato sono disponibili molti prodotti che favoriscono il debridement autolitico, reidratano ed ammorbidiscono l'escara favorendo il suo rigonfiamento facilitandone il distacco; si utilizzano a tale scopo idrogeli, idrocolloidi, medicazioni idroreattive, idrofibre e medicazioni multicomponenti, in base alla quantità e alla qualità del tessuto necrotico.

Lo sbrigliamento meccanico è basato sull'utilizzo di medicazioni a base di garze asciutte, garze bagnatoasciutte (wet to dry), garze impregnate/paraffinate, o una medicazione in fibre monofilamento per rimuovere il tessuto non vitale dal letto della ferita; può causare sanguinamento e dolore.

Lo sbrigliamento chirurgico, denominato anche sharp (bisturi) è una tecnica più invasiva, indicata in caso di ferite infette e nel caso in cui vi sia presente un'elevata quantità di tessuto necrotico o devitalizzato. Comprende procedure chirurgiche minori, con rimozione di tessuto mediante bisturi o forbici, fino a procedure chirurgiche complesse eseguite in anestesia generale, che richiedono la presenza di un operatore sanitario specializzato che sappia gestire eventuali complicazioni legate al sanguinamento. ${ }^{16}$

Il debridement enzimatico è un metodo più lento e maggiormente selettivo rispetto agli altri ed è mirato alla degradazione del collagene tissutale attraverso l'impiego di un enzima proteolitico denominato collagenasi. Si tratta di prodotti sicuri e di manipolazione facile che non determinano l'insorgenza di dolore ma questo metodo non può essere utilizzato per ferite con un elevato grado di infezione e non può essere associato a medicazioni a base di argento. ${ }^{34}$

Il debridement biologico viene anche denominato terapia larvale poiché prevede l'impiego di larve sterili della specie Lucilla sericata in grado di secernere enzimi che digeriscono il tessuto necrotico e impediscono la ricrescita del biofilm batterico senza ledere il tessuto di granulazione. ${ }^{35}$

Infine, sono necessari materiali di copertura della lesione che abbiano caratteristiche di biocompatibilità, mantengano un microambiente umido, promuovano il processo di riparazione tissutale evitando la macerazione della pelle circostante e proteggano da infezioni esogene. Per scegliere la tipologia di medicazione più adatta al trat- 
tamento della lesione vengono valutate la quantità e la qualità di essudato, ${ }^{36}$ la presenza di infezione o eventuale rischio infettivo, le dimensioni della ferita e la tipologia di debridement praticata. L'obiettivo, infatti, è quello di favorire la rimozione di essudato e materiale necrotico, conservando integra la cute perilesionale e mantenendo un microambiente umido, una temperatura costante e un equilibrio tra la permeabilità utile allo scambio dei gas e barriera protettiva rispetto a microrganismi esterni. La medicazione deve ovviamente essere conformabile e adattabile alle superficie irregolari e ai contorni anatomici ed essere maneggevole, confortevole e tollerabile per il paziente; deve essere atraumatica al cambio, per ridurre il dolore, e deve consentire tempi di cambio più lunghi, per ridurre il disagio per il paziente e il caregiver. Infine, è necessario che siano disponibili dispositivi sostenibili, con costi non eccessivi per garantire una equità di cura.

È possibile proporre una classificazione funzionale delle medicazioni si basa sulla capacità dell'operatore sanitario di riconoscere il segno prevalente, cioè il segno clinico più evidente all'esame obiettivo della lesione e della cute perilesionale, che identifica il tempo clinico della lesione e orienta l'operatore verso la scelta della medicazione più opportuna in quel determinato momento. La classificazione funzionale ${ }^{37}$ delle medicazioni comprende: i) Medicazioni favorenti autolisi e sbrigliamento; quando sia necessario rimuovere, ridurre o lisare il tessuto non vitale attraverso l'apporto idrico o l'azione osmotica (idrogeli amorfi, idrocolloidi in placca e in pasta, miele di manuka) oppure attraverso l'attività enzimatica (enzimi proteolitici); ii) Medicazioni favorenti la granulazione; in occlusione (idrocolloidi in placca), per assorbimento (schiume in poliuretano, fibra di cellulosa, alginato di $\mathrm{Ca} / \mathrm{Na}$, terapia a pressione negativa) biointerattive (con collagene o con acido ialuronico); iii) Medicazioni antimicrobiche, semplici o complesse. Queste ultime possono essere adsorbenti (poliuretano + argento), ritentive (fibre cellulosa + argento), emostatiche (alginati + argento), favorenti il debridement (cadexomero, miele di manuka) o bioattive (collagene + argento); iv) Medicazioni riepitelizzanti eudermiche; protettive atraumatiche (garze lipidocolloidali, garze siliconate, garze vaselinate) , mantenenti l'umidità (idrocolloidi, film in poliuretano) o preventive di macerazione e/o lenitive (bende a base di ossido di zinco)

\section{IMPATTO DELLA LESIONE SULLA QUALITÀ DI VITA (QOL)}

La presenza di una lesione cutanea cronica può incidere in elevata misura sulla qualità di vita (Quality of Life, QoL) del paziente ed influenzare gli outcome clinici. Diversi aspetti della vita quotidiana vengono influenzati e modificati dalla presenza di una ferita cronica, come la vita famigliare e sociale, la vita lavorativa e la percezione del sé soprattutto nel sesso femminile; possono insorgere difficoltà nei rapporti interpersonali a causa dell'aspetto o dell'odore della ferita e limitazioni funzionali, isolamento, disabilità e depressione che influenzano negativamente la $\mathrm{QoL}^{38}$ e impongono un approccio diagnostico e terapeutico della ferita cronica che sia quanto più aderente alle esigenze individuali. ${ }^{39}$ In quest'ottica il management della lesione cutanea cronica deve essere multidisciplinare e coinvolgere più figure sanitarie oltre al paziente e il $c a-$ regiver al fine di migliorare il patient journey. La formazione e l'aggiornamento del personale sanitario, in modo particolare degli infermieri dedicati all'assistenza domiciliare territoriale, in tema di wound care è fondamentale dato il suo coinvolgimento non solo nel trattamento della lesione e nell'eliminazione dei fattori che possono influire negativamente sulla guarigione ma anche sulla compliance del paziente e nei percorsi di engagement ed empowerment del paziente e dei familiari. ${ }^{40}$

L'intero processo di cura, a partire dall'accertamento diagnostico fino al follow up, dovrebbe prevedere una gestione integrata tra Medico di Medicina Generale (MMG), caregiver e personale infermieristico per attuare un'assistenza continua, personalizzata, basata sulle evidenze scientifiche e inclusiva di percorsi educativi.

\section{TELEMEDICINA: UNA MODALITÀ INNOVATIVA PER LA GESTIONE DEL WOUND CARE}

Secondo il recente documento del Governo Italiano Conferenza Permanete per i rapporti tra lo Stato le Regioni e le Province autonome di Trento e Bolzano del 27/10/2020 "la Telemedicina rappresenta un approccio innovativo alla pratica sanitaria consentendo l'erogazione di servizi a distanza attraverso l'uso di dispositivi digitali, internet, software, e delle reti di telecomunicazione. Attraverso la telemedicina è possibile garantire la fruizione di servizi sanitari senza che il paziente o l'assistito debba recarsi presso le strutture sanitarie, rendendo accessibili le cure [...]".

La telemedicina rappresenta oggi un campo in continua evoluzione ed espansione con molteplici vantaggi, tra cui il miglioramento delle tempistiche di intervento con riduzione dei tempi d'attesa, l'abbattimento dei costi relativi alla gestione ambulatoriale delle visite e, in era COVID-19 $9^{41}$ rappresenta anche una risorsa importante per l'erogazione delle prestazioni sanitarie in completa sicurezza. $^{42}$

L'esperienza degli Autori nel territorio Ligure, durante la pandemia COVID-19, ha messo in luce alcuni punti importanti nel management delle lesioni croniche mediante l'uso della telemedicina ${ }^{43}$ che come confermato anche da alcuni studi presenti in letteratura, ${ }^{44-47}$ affianca ed integra efficacemente il percorso clinico tradizionale senza sosti- 
tuirlo del tutto. Secondo gli esperti, infatti, rimane imprescindibile e di fondamentale importanza effettuare la prima visita in presenza al fine di ottimizzare il percorso diagnostico ed il successivo follow up .

L'impiego degli strumenti dell'Information Technology e della Telemedicina consentono di seguire i pazienti a distanza, dal loro domicilio, utilizzando computer, tablet e smartphone con applicazioni specificamente ideate per la gestione delle immagini della ferita e dei dati clinici salienti del paziente. Gli outcome sembrano confermare inoltre, che la tecnologia è ben accolta dal paziente ${ }^{48,49}$ con un miglioramento dell'aderenza al piano di cura. Persistono tuttavia alcune limitazioni all'uso delle tecnologie digitali nel wound care; in alcune aree geografiche i pazienti non hanno accesso alle infrastrutture necessarie (ad esempio, dispositivi video abilitati o internet ad alta velocità) per partecipare alla televisita e questo impedisce o rende complicata una corretta valutazione a distanza dello stato del paziente e della ferita. Anche l'invio dell'immagine della lesione allo specialista può presentare dei limiti quando effettuata dal paziente, caregiver o anche da un sanitario non correttamente formato. ${ }^{50}$ Altro aspetto fondamentale per l'erogazione delle prestazioni di telemedicina è la digitalizzazione del sistema attraverso una strumentazione in grado di supportare elevate qualità di immagini e connessioni veloci. ${ }^{51}$ La valutazione diagnostica dello stato di una lesione necessita infatti di un'alta risoluzione d'immagine (pixel) e di una connessione in grado di consentire il download di file di grandi dimensioni. Tali requisiti devono essere assicurati per rendere possibile una prestazione sanitaria adeguata in via telematica. ${ }^{52}$

L'educazione del caregiver e del personale sanitario all'uso corretto di adeguati strumenti digitali per il management delle ferite croniche rappresenta perciò una priorità per il corretto utilizzo della telemedicina e delle nuove tecnologie di intelligenza artificiale.

Alcuni studi hanno evidenziato anche i vantaggi sui processi assistenziali e organizzativi, anche in termini di costi, applicando la telemedicina al management delle lesioni croniche. Oltre alla protezione del paziente in fase pandemica, garantita dalla riduzione degli accessi in ospedale, pazienti fragili con ridotta mobilità e famiglie fragili possono evitare spostamenti. I benefici derivano anche dalla diminuzione degli spostamenti del personale, soprattutto in aree impervie, riduzione del tempo non produttivo delle risorse dedicate al wound care e maggiore efficienza nell'organizzazione degli appuntamenti. Trasportare questo tipo di innovazione all'interno dei sistemi socio-sanitari, richiede un coordinamento territoriale, un approccio interdisciplinare, la dotazione di strumenti adeguati, la codifica delle prestazione erogate, il rispetto della privacy del paziente e più in generale la diffusione di un cambiamento culturale che richiederà tempo e impegno..$^{53}$

\section{CONCLUSIONI}

Le lesioni cutanee croniche rappresentano una patologia con un trend in costante aumento dato il progressivo invecchiamento della popolazione. L'impatto socio-sanitario ed economico di questa patologia è elevato ed influenza la compliance del paziente in termini di reperibilità e costi dei presidi medici e in termini di gestione della ferita da parte del caregiver che deve essere formato a tale scopo. La presenza di un team multidisciplinare (medico di medicina generale, infermiere territoriale della assistenza domiciliare integrata, specialisti ospedalieri) in grado di coordinare l'iter terapeutico dalla diagnosi al follow up è di fondamentale importanza nella gestione delle ulcere croniche e può condurre ad un abbattimento dei costi del SSN e ad una migliore interazione medico-paziente.

Per la risoluzione delle ferite croniche è indispensabile agire su tutti i fattori che ne rallentano la guarigione, agendo efficacemente sul microambiente di ferita, riducendo la carica batterica attraverso una corretta detersione e antisepsi e utilizzando medicazioni avanzate di semplice utilizzo e durature, sicure per il paziente e la cui rimozione non sia dolorosa.

Alla luce delle recenti esperienze la Telemedicina sembra fornire una valida opzione per il management a distanza del paziente con ferite croniche sebbene sia ancora necessaria una sua implementazione.

\section{BIBLIOGRAFIA}

1. Graves N, Phillips CJ, Harding K. A narrative review of the epidemiology and economics of chronic wounds. Br J Dermatol Online ahead of print, 2021; doi:10.1111/bjd.20692

2. ISTAT. Rapporto annuale 2019. ISTAT, 2019. Disponibile presso: https://www.istat.it/storage/rapporto-annuale/2019/ Sintesi2019.

3. Rayala BZ. Skin ulcers: Prevention and diagnosis of pressure, venous leg, and arterial ulcers. FP Essentials 2020;499:11-8.

4. Kirsner RS, Vivas AC. Lower-extremity ulcers: Diagnosis and management. Br J Dermatol 2015;173:13953.

5. Zaidi SRH, Sharma S. Decubitus ulcer. StatPearls. Published 2021. PMID: 31971747.

6. Reddy M, Gill SS, Kalkar SR, et al. Treatment of pressure ulcers. JAMA 2008;300:2647.

7. Boyko T V., Longaker MT, Yang GP. Review of the current management of pressure ulcers. Adv Wound Care 2018;7: 57-67.

8. Commissione Regionale Dispositivi Medici Regione EmiliaRomagna. Le medicazioni avanzate per il trattamento delle ferite acute e croniche. Commissione Regionale Dispositivi Medici Regione Emilia-Romagna, 2016. Disponibile presso: http://salute.regione.emilia-romagna.it/farmaci/dispositivimedici

9. Ratliff CR, Yates S, McNichol L, Gray M. Compression for primary prevention, treatment, and prevention of recurrence 
of venous leg ulcers. J Wound Ostomy Cont Nurs 2016;43: 347-64.

10. Lim SLX, Chung RE, Holloway S, Harding KG. Modified compression therapy in mixed arterial-venous leg ulcers: An integrative review. Int Wound J Online ahead of print, 2021. doi:10.1111/iwj.13585

11. Alexiadou K, Doupis J. Management of diabetic foot ulcers. Diab Ther 2012;3:4.

12. Abbott CA, Carrington AL, Ashe H, et al. The north-west diabetes foot care study: Incidence of, and risk factors for, new diabetic foot ulceration in a community-based patient cohort. Diabetic Med 2002;19:377-84.

13. Lauterbach S, Kostev K, Kohlmann T. Prevalence of diabetic foot syndrome and its risk factors in the UK. J Wound Care 2010;19:333-7.

14. Doupis J, Veves A. Classification, diagnosis, and treatment of diabetic foot ulcers. Wounds 2008;20:117-26.

15. Anichini R, Brocco E, Cavallini M, et al. Grey-zone nel trattamento del paziente con Piede Diabetico. I risultati di una Delphi survey italiana multidisciplinare condivisa tra esperti. Ital J Wound Care 2021;4:56.

16. Flanagan M. Wound healing and skin integrity: Principles and practice. Wiley-Blackwel; 2013.

17. Schultz GS, Sibbald RG, Falanga V, et al. Wound bed preparation: A systematic approach to wound management. Wound Repair Regen 2003;11:S1-28.

18. Greener B, Hughes AA, Bannister NP, Douglass J. Proteases and $\mathrm{pH}$ in chronic wounds. J Wound Care 2005;14:59-61.

19. Bottos S, Tasinato R, Silvestrini S. Wound Care. Un moderno approccio alla gestione delle lesioni cutanee. Effetti Editore, ed. ReadFiles 2019; Supplement 4:1-22.

20. Roberts CD, Leaper DJ, Assadian O. The role of topical antiseptic agents within antimicrobial stewardship strategies for prevention and treatment of surgical site and chronic open wound infection. Adv Wound Care 2017;6:63-71.

21. Lipsky BA, Hoey C. Topical antimicrobial therapy for treating chronic wounds. Clin Infect Dis 2009;49:1541-9.

22. Lipsky BA, Dryden M, Gottrup F, et al. Antimicrobial stewardship in wound care: A position paper from the British Society For Antimicrobial Chemotherapy and European Wound Management Association. J Antimicrob Chemother 2016;71: 3026-35.

23. Cooper R. Meeting report: antimicrobial stewardship in wound management. Wounds Int 2017;8:46-47.

24. Williamson DA, Carter GP, Howden BP. Current and emerging topical antibacterials and antiseptics: Agents, action, and resistance patterns. Clin Microbiol Rev 2017;30:827-60.

25. Scalise A. La nuova formulazione di soluzione elettrolitica di sodio ipoclorito allo $0,05 \%$ soluzione cutanea: razionale e vantaggi nel trattamento delle lesioni infette. AboutOpen Dermatol 2021;8:14-22.

26. Kramer A, Dissemond J, Kim S, et al. Consensus on wound antisepsis: Update 2018. Skin Pharmacol Physiol 2018;31: 28-58.

27. Bigliardi PL, Alsagoff SAL, El-Kafrawi HY, et al. Povidone iodine in wound healing: A review of current concepts and practices. Int J Surg 2017;44:260-8.

28. Cassino R, Ippolito AM. Chloroxidating solution vs. superoxide solution: a comparison in wound cleansing. Acta Vulnologica 2012;10:143-52.
29. Santo G, D'atanasio N, Capezzone De Joannon A, et al. Valutazione della attività e tossicità di una soluzione di ipoclorito di sodio elettrolitico allo $0,05 \%$ nel processo di riparazione tissutale. Gazzetta Medica Italiana 2012;171:795-805.

30. Aliquò MS, Monge L, Romagnoli F, Tasinato R. Trattamento delle ulcere croniche difficili: il ruolo centrale della preparazione del letto della ferita. AboutOpen 2017;3:43-62.

31. Dowsett C, Newton H. Wound bed preparation: TIME in practice. Wounds UK 2005; 1:58.

32. Stephen-Haynes J, Thompson G. The different methods of wound debridement. Br J Comm Nurs 2007;12:S6, S8-10, S12-14, S16.

33. Strohal R, Dissemond J, Jordan O'Brien J, et al. EWMA document: Debridement: An updated overview and clarification of the principle role of debridement. J Wound Care 2013;22:5.

34. Schultz G, Ladwig G, Wysocki A. Extracellular matrix: Review of its roles in acute and chronic wounds. World Wide Wounds 2005;2005.

35. Thomas AM, Harding KG, Moore K. The structure and composition of chronic wound eschar. J Wound Care 1999;8:285-7.

36. Britto EJ, Nezwek TA, Robins M. Wound Dressings. In: StatPearls 2021.

37. AIUC. Documento di posizionamento. Medicazione dell'ulcera cutanea. Acta vulnologica vol 12-3.

38. Silva DC da, Budó MDLD, Schimith MD et al. Experiências Construídas No Processo De Viver Com A Úlcera Venosa. Cogitare Enfermagem 2015;20:37784.

39. Szewczyk MT, Mościcka P, Jawień A, et al. Quality of life in patients with leg ulcers or skin lesions - A pilot study. Adv Dermatol Allergol 2015;6:465-9.

40. Dhar A, Needham J, Gibb M, Coyne E. The outcomes and experience of people receiving community-based nurse-led wound care: A systematic review. J Clinical Nurs 2020;29: 2820-33.

41. Istituto Superiore di Sanità. Indicazioni ad interim per servizi assistenziali di telemedicina durante l'emergenza sanitaria COVID-19. Istituto Superiore di Sanità, 2020.

42. Mills EC, Savage E, Lieder J, Chiu ES. Telemedicine and the COVID-19 pandemic: Are we ready to go live? Adv Skin Wound Care 2020;33:410-7.

43. Cannavale T, Nocco G, Cozzani E, et al. Managing telemedicine for wounds in the COVID-19 era: A new proposal. Adv Skin Wound Care 2021;34:120.

44. Bolton L. Remote wound care. Wounds 2020;32:350-2.

45. Huang Z, Wu S, Yu T, Hu A. Efficacy of telemedicine for patients with chronic wounds: A meta-analysis of randomized controlled trials. Adv Wound Care 2021;10:103-112.

46. Nordheim LV, Haavind MT, Iversen MM. Effect of telemedicine follow-up care of leg and foot ulcers: A systematic review. BMC Health Serv Res 2014;14:565.

47. Sood A, Granick MS, Trial C, et al. The role of telemedicine in wound care: A review and analysis of a database of 5,795 patients from a mobile wound-healing center in LanguedocRoussillon, France. Plastic Reconstr Surg 2016;138:248S$256 \mathrm{~S}$.

48. Dobke MK, Bhavsar D, Gosman A, et al. Pilot trial of telemedicine as a decision aid for patients with chronic wounds. Telemed J E-Health 2008;14:245-9. 
49. Bergquist TF, Thompson K, Gehl C, Munoz Pineda J. Satisfaction ratings after receiving internet-based cognitive rehabilitation in persons with memory impairments after severe acquired brain injury. Telemed J E-Health 2010;16: 417-23.

50. Dobke MK, Bhavsar D, Herrera F. Do telemedicine wound care specialist consults meet the needs of the referring physician? A survey of primary care providers. Int $\mathrm{J}$ Telemed Appl Online ahead of print 2011, doi:10.1155/ 2011/321376
51. Jones SM, Banwell PE, Shakespeare PG. Telemedicine in wound healing. Int Wound J 2004;1:225-30.

52. Liu L, Wang L, Huang Q, et al. An efficient architecture for medical high-resolution images transmission in mobile telemedicine systems. Computer Methods Programs Biomed 2020;187:105088.

53. Scalise A, Falcone M, Avruscio G, et al. What COVID-19 taught us: new opportunities and pathways from telemedicine and novel antiseptics in wound healing. Int Wound J Online ahead of print 2021, doi:10.1111/iwj.13695 